\title{
SELFSENSING CONTROL OF PMSM AT ZERO AND LOW SPEED
}

The paper deals with control of Permanent Magnet Synchronous Motor (PMSM) without a rotor position sensor. In the low and zero speed region, the rotor saliencies are utilized to obtain the information about the rotor position. Self-sensing control techniques employ high frequency (hf) signal injection to excite the saliencies. The proposed algorithm enables tracking the saliency and allows PMSM control at zero and low speed. The theoretical assumptions were experimentally verified including the initial rotor position detection.

Keywords: PMSM, self-sensing, low speed, position estimation, hf signal injection

\section{Introduction}

Vector control of AC machines like FOC (Field Oriented Control) or DTC (Direct torque control) provides a very good dynamic performance and full start-up torque. This technique enables separate control of electromagnetic torque and magnetizing flux of the motor. On the other hand, these structures require information about the rotor position. This can be obtained by mechanical sensors such as Hall Effect sensors, encoders or resolvers. A control without these mechanical sensors is known as self-sensing, auto-sensing or eigen-sensing since the position information is an inherent part of the machine thanks to magnetic and construction asymmetry ('the machine is the sensor') [1-3] It brings benefits such as lower cost of the drive (which is an important factor in automotive industry), capability of high speed operation (where sensor is mechanical restriction) and can be used as redundant solution in case of sensor failure. There are two categories of these algorithms: model based methods and methods utilizing the machine anisotropy that is a function of the rotor position. As an example of model based method, a backEMF observer can be mentioned. However, this observer can be used only for medium to high speed operating range because the amplitude of back-EMF voltage is proportional to the angular velocity and is very small for precise position estimation at low speed.

The significant saliency can be observed in interior PM synchronous machine, which yields to different inductance in $\mathrm{d}$ and q-axis. This paper describes a saliency tracking method to estimate the rotor position. The magnetic saliency is excited with the high frequency $(h f)$ voltage signal. Proposed algorithm has been designed to work at zero and low speed region, including detection of initial rotor position. This algorithm together with a model based observer $[4,5]$ offers a solution for PMSM control without a position sensor within the entire speed range.

\section{PMSM mathematical model}

To simplify mathematical model of 3-phase machine, 2-phase rotational $(d q)$ frame can be used, which is aligned and rotates synchronously with the rotor. After transformation of voltage and torque equations and some mathematical corrections, motor model can be written as follows:

$\left[\begin{array}{l}u_{d} \\ u_{q}\end{array}\right]=R_{s}\left[\begin{array}{l}i_{d} \\ i_{q}\end{array}\right]+\left[\begin{array}{cc}L_{d} & 0 \\ 0 & L_{q}\end{array}\right] \frac{d}{d t}\left[\begin{array}{c}i_{d} \\ i_{q}\end{array}\right]+\omega_{e}\left[\begin{array}{c}-L_{q} \\ L_{d}\end{array}\right]\left[\begin{array}{c}i_{d} \\ i_{q}\end{array}\right]+\omega_{e} \psi_{p m}\left[\begin{array}{l}0 \\ 1\end{array}\right](1)$

where $R_{s}$ is stator resistance, $L_{d}$ and $L_{q}$ are $\mathrm{d}$ and $\mathrm{q}$ axis synchronous inductances, $\omega_{e}$ is electric angular speed and $\psi_{p m}$ is flux of permanent magnets.

$m_{e}=\frac{3}{2} \cdot p \cdot\left(\psi_{p m} \cdot i_{q}+\left(L_{d}-L_{q}\right) \cdot i_{d} \cdot i_{q}\right)$

In Equation (2), the first synchronous electromagnetic torque term is independent of $i_{d}$ current, but it is directly proportional to the stator current component $i_{q}$. In contrast, the second reluctance torque term is proportional to the $i_{d} i_{q}$ current component product and to the difference of the inductance values

\footnotetext{
* ${ }^{1}$ Simon Zossak, ${ }^{2}$ Marek Stulrajter, ${ }^{1}$ Pavol Makys

${ }^{1}$ Department of Power Electrical Systems, Faculty of Electrical Engineering, University of Zilina, Slovakia

${ }^{2}$ NXP Semiconductors, Roznov pod Radhostem, Czech Republic

E-mail: simon.zossak@fel.uniza.sk
} 


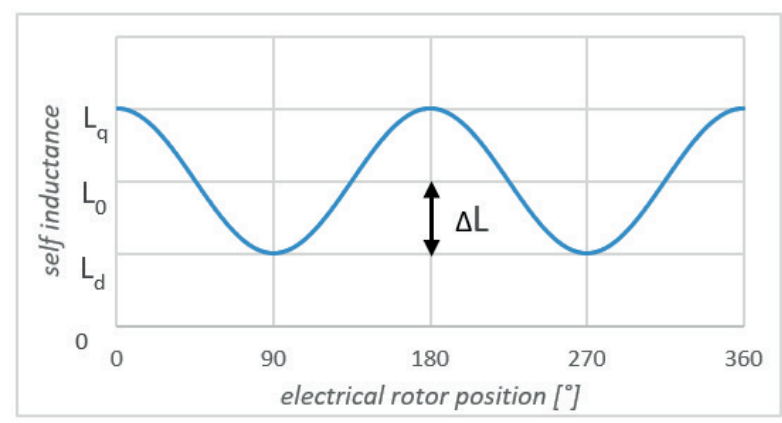

Figure 1 Stator phase self-inductance as a function of rotor position

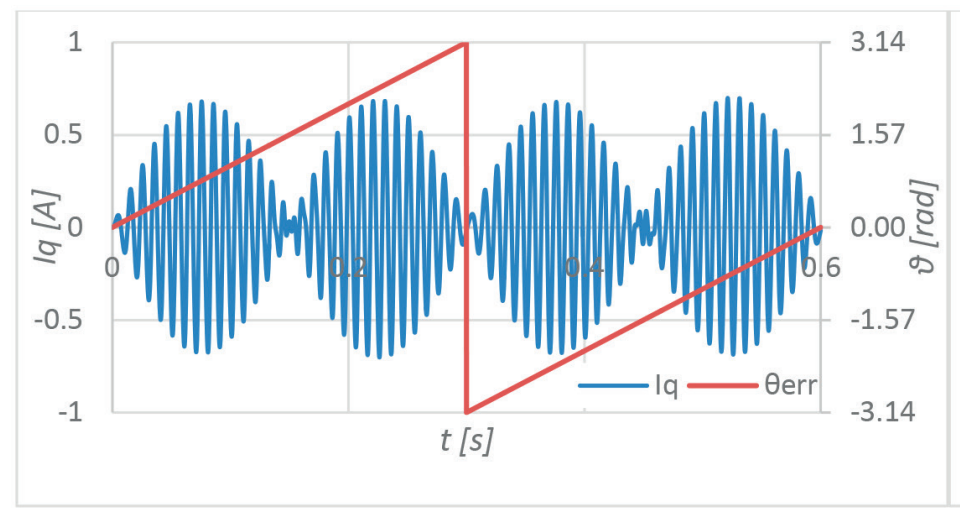

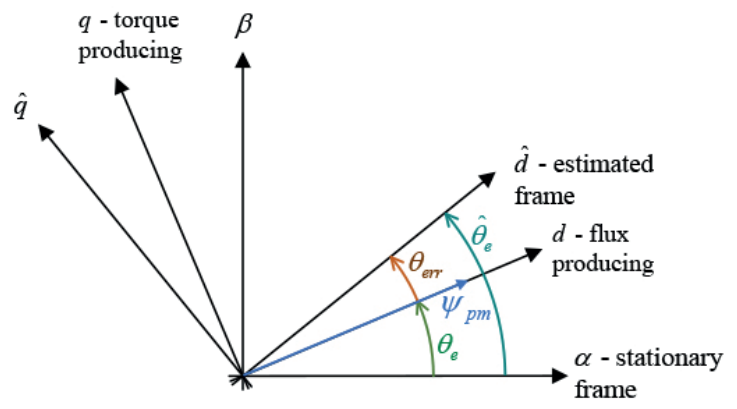

Figure 2 Estimated and real reference frame

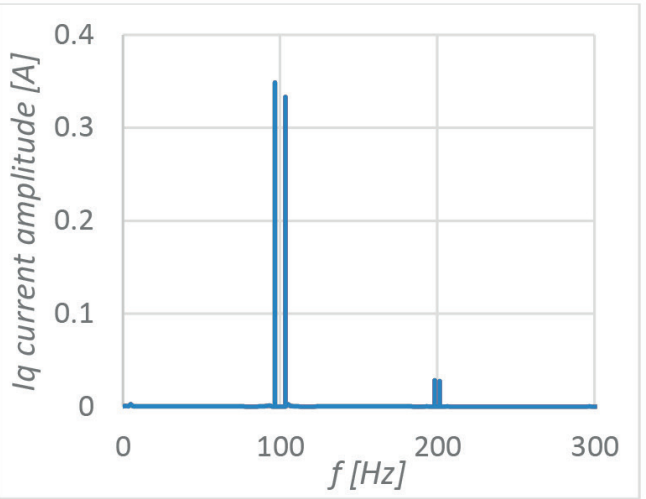

Figure 3 Dependence of current amplitude on estimation error $\theta_{\text {err }}$ and frequency spectrum of $\hat{i}_{q}$

$\left(L_{d}-L_{q}\right)$. The angular speed is proportional to the difference between electromagnetic and load torque [6-8]:

$$
\frac{d \omega_{e}}{d t}=\frac{p}{J}\left(m_{e}-m_{z t}\right)
$$

The inductance variation in salient-pole machines arises because the permanent magnets are either inset or buried in the rotor. Since the permeability of the permanent magnets is low $\mu_{r M} \approx \mu_{0}$, the effective air-gap length is larger in the direction of the magnets flux than it is in perpendicular direction. Magnetic reluctance in d-axis is therefore high and inductance low. On the contrary, magnetic reluctance in q-axis is low and inductance high. So, the inequality $L_{d}>L_{q}$ applies.

This type of the motor saliency is directly linked to the rotor position. The stator phase inductance as function of the rotor position is shown in Figure 1.

To track this saliency and thus extract the rotor position information, an additional signal needs to be added to the supply voltage. There is a plenty of methods using different approach to excite the motor. The most common are injection of high frequency sinusoidal voltage signal or discrete voltage test pulses (e.g. INFORM method). Such signal excites the magnetic saliency and enables its extraction. The high frequency voltage signal injection is used in this paper, due to the easier implementation with voltage source inverter [6,9].
To analyze the motor behavior in high frequency spectra, the mathematical model of PMSM described in Equation (1) is transformed to frequency domain:

$$
\begin{aligned}
& {\left[\begin{array}{l}
u_{d}\left(j \omega_{c}\right) \\
u_{q}\left(j \omega_{c}\right)
\end{array}\right]=\left[\begin{array}{cc}
R_{s}+j \omega_{c} L_{d} & -\omega_{e} L_{q} \\
\omega_{e} L_{q} & R_{s}+j \omega_{c} L_{d}
\end{array}\right]\left[\begin{array}{c}
i_{d}\left(j \omega_{c}\right) \\
i_{q}\left(j \omega_{c}\right)
\end{array}\right]+} \\
& +\omega_{e}\left[\begin{array}{c}
0 \\
\psi_{p m}
\end{array}\right]
\end{aligned}
$$

where $\omega_{c}$ is frequency of injected signal, that is much higher than fundamental frequency of the motor $\omega_{e}\left(\omega_{c}>\omega_{e}\right)$.

Assuming the $h f$ voltage signal is injected only in estimated d-axis, then $\hat{u}_{d}=U_{m} \sin \left(\omega_{c} t\right)$ and $\hat{u}_{q}=0$, and current components can be expressed as follows [4]:

$\left[\begin{array}{c}\hat{i}_{d} \\ \hat{i}_{q}\end{array}\right]=-\frac{\hat{U}_{m}}{\omega_{c} L_{d} L_{q}} \cos \left(\omega_{c} t\right)\left[\begin{array}{c}L_{0}-\Delta L \cos \left(2 \theta_{\text {err }}\right) \\ \Delta L \sin \left(2 \theta_{\text {err }}\right)\end{array}\right]$

where angle $\theta_{\text {err }}$ is angle between estimated and real reference frame:

$\theta_{e r r}=\hat{\theta}_{e}-\theta_{e}$

The position of real reference frame is given by the position of permanent magnet flux and the position of estimated reference frame is given by the estimated position of rotor flux as shown in Figure 2. 


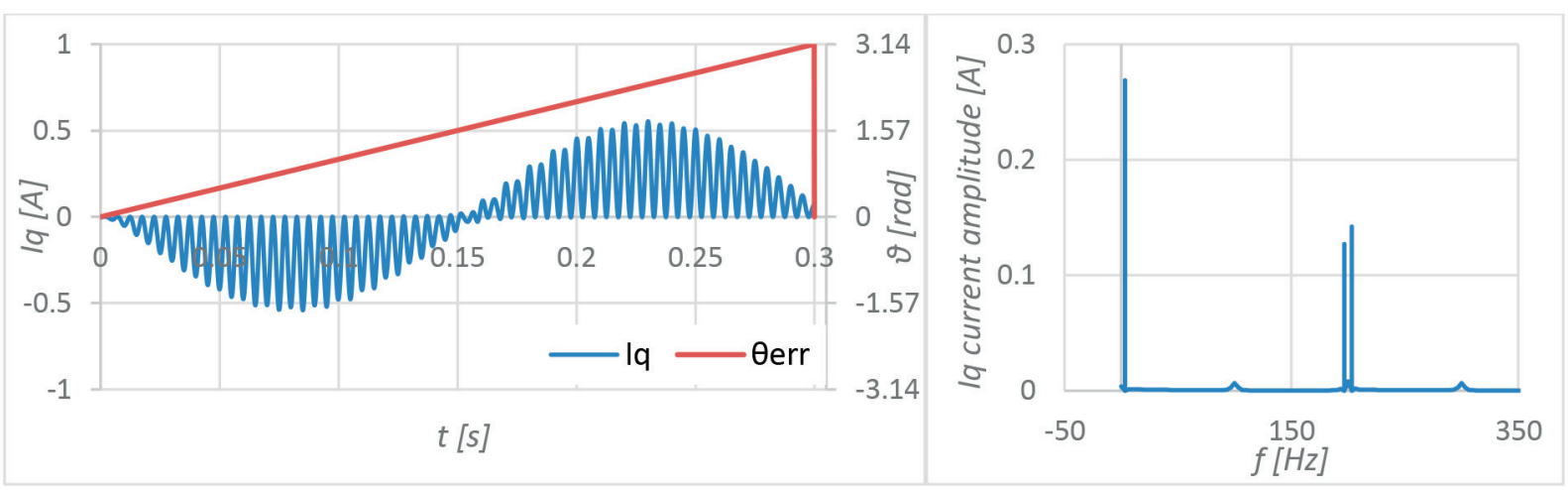

Figure 4 Demodulated current $\hat{i}_{q \_d m d}$ and its frequency spectrum

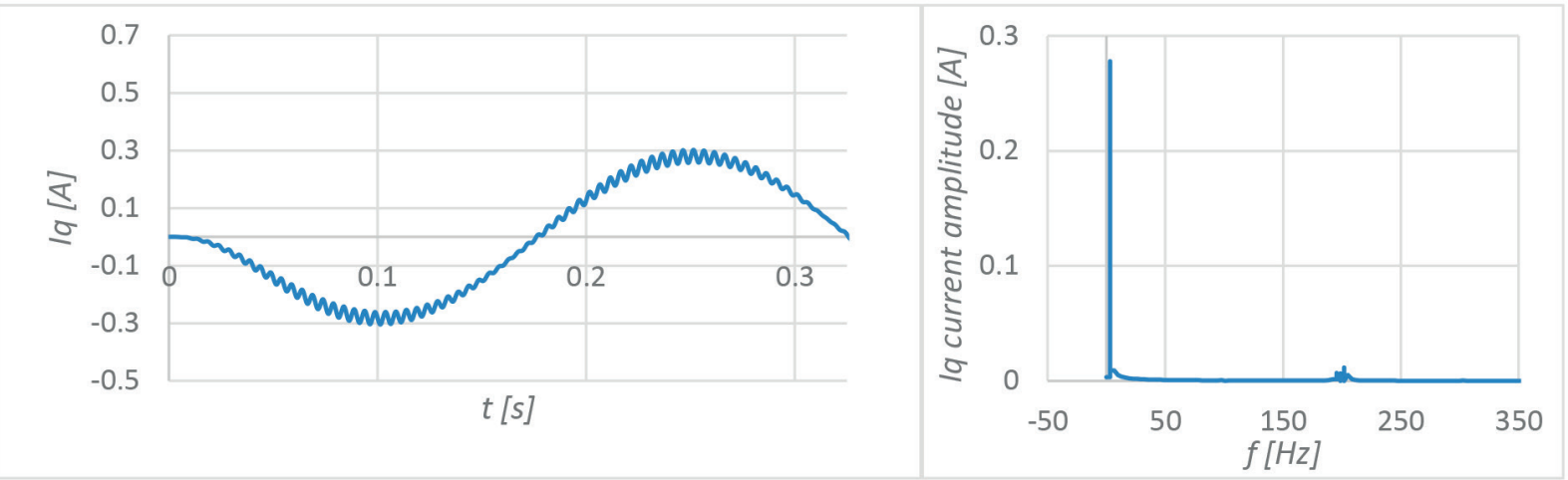

Figure 5 Filtered current $\hat{i}_{q_{-} L P F}$ and its frequency spectrum

\section{Saliency observer}

The position of permanent magnet flux (d-axis) needs to be known for maximal efficiency of FOC. The angle $\theta_{\text {err }}$ can be extracted from the Equation (5) for current $\hat{i}_{q}$. The waveform of this current during one electrical revolution of the rotor is shown in Figure 3. The current has zero amplitude if the angle $\theta_{\text {err }}$ is equal to any integer multiple of $\frac{\pi}{2}$.

The high and low frequency components are separated by heterodyning demodulation. Demodulation is based on multiplication of the current $\hat{i}_{q}$ from Equation (5) by an auxiliary signal $\cos \left(\omega_{c} t\right)$ with identical frequency and phase as modulated signal [4]:

$\hat{i}_{q}=-\frac{\hat{U}_{m} \Delta L}{\omega_{c} L_{d} L_{q}} \cos \left(\omega_{c} t\right) \sin \left(2 \theta_{\text {err }}\right) \quad / \cdot \cos \left(\omega_{c} t\right)$

By applying the mathematical rules, the Equation (7) will be transformed into the following form:

$\hat{i}_{q_{-} d m d}=-\frac{I_{1}}{2} \sin \left(2 \theta_{\text {err }}\right)-\frac{I_{1}}{2} \sin \left(2 \theta_{\text {err }}\right) \cos \left(2 \omega_{c} t\right)$

where the magnitude of the original and modulated current is expressed as:

$$
I_{1}=-\frac{\hat{U}_{m} \Delta L}{\omega_{c} L_{d} L_{q}}
$$

The low and high frequency component are clearly visible in spectrum of demodulated current in Figure 4.

High frequency component in Equation (8) can be afterwards attenuated with a Low Pass Filter:

$\hat{i}_{q \_L P F}=-\frac{I_{1}}{2} \sin \left(2 \theta_{\text {err }}\right)$

The current waveform after filtering the high frequency component can be seen in Figure 5 .

The angle $\theta_{\text {err }}$ can then be calculated as follows:

$\theta_{\text {err }}=-\frac{1}{2} \arcsin \left(\frac{-2 \cdot \hat{i}_{q L P F}}{I_{1}}\right)$

Saliency observer [4] is in fact a closed-loop system which forces the filtered component of the current $\hat{i}_{q}$ to be zero, and therefore setting $\theta_{\text {err }}$ to zero. Thus the estimated $d q$ frame match the real $d q$ frame, and estimated rotor position reflex the rotor flux position.

The control structure of saliency observer is shown in Figure 6 . The abc currents are transformed to the estimated rotational frame $\hat{d} \hat{q}$ using the estimated rotor position. 


\section{kom Nikacie}

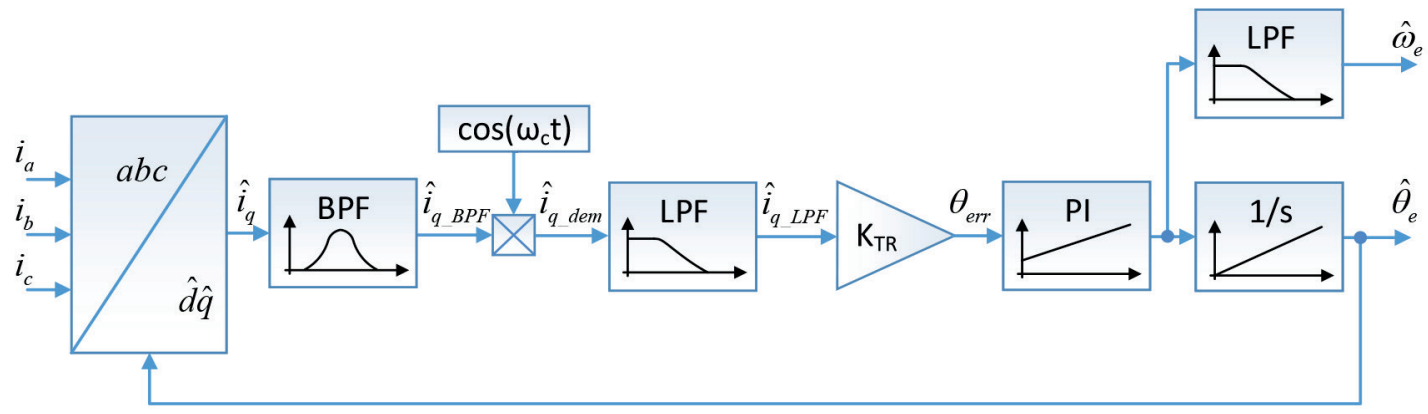

Figure 6 Block diagram of Saliency observer
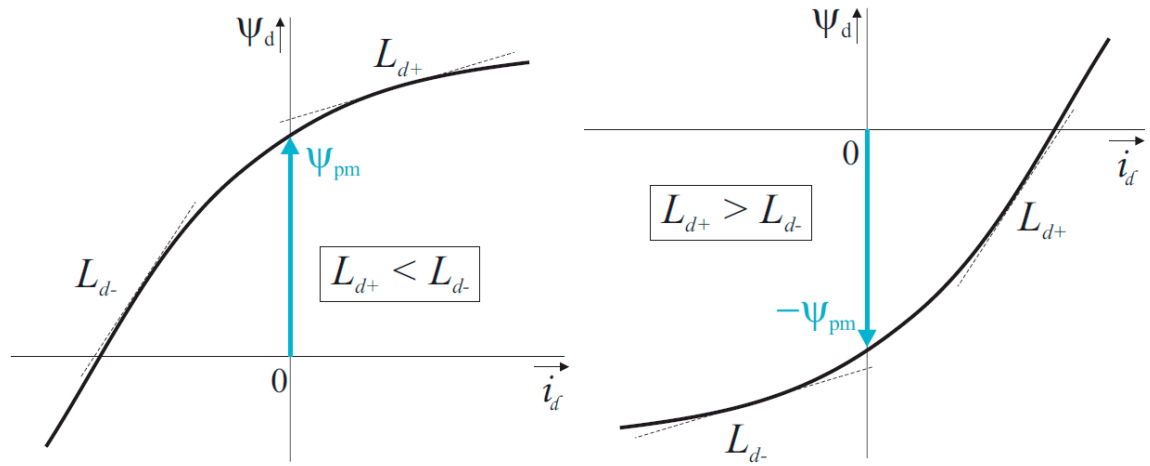

Figure 7 Magnetic flux in d-axis as function of $i_{d}$ current, corresponding to north (on the left) and south (on the right) pole

The current $\hat{i}_{q}$ is used for demodulation by heterodyning and set of filters, to extract the information about the error angle between the real $d q$ frame and estimated $d q$ frame. The estimation error $\theta_{\text {err }}$ is then processed by a PLL to generate the estimated rotor position by minimizing the estimation error.

\section{PM polarity detection}

As can be seen in Equation (10), the term of current $\hat{i}_{q L P F}$ can be zero if the estimation error is $\theta_{\text {err }}=k \cdot 2 \pi$, but also if it is $\theta_{\text {err }}=\pi+k \cdot 2 \pi$, where $k=0, \pm 1 . \pm 2, \ldots$. The $\pi$ offset in the position error indicates a displacement $180^{\circ}$ between the north and south pole of the magnet. To eliminate this phenomenon, an initial position detection approach including the PM polarity detection has to be adopted. The PM polarity detection is based on stator core saturation phenomenon. Assuming the magnetic operating point is placed on the knee of the hysteresis curve, making the saturation effect visible even with small variation in d-axis current $\hat{i}_{d}$ as shown in Figure 7 .

This theoretical relationship can be approximated using Taylor series expansion as follows [4]:

$$
\begin{aligned}
& i_{d_{\_} \text {north }} \approx \frac{1}{L_{d}}\left(\psi_{d}-\psi_{p m}\right)-\frac{1}{2} \frac{d^{2} i_{d}}{d \psi_{d}^{2}}\left(\psi_{d}-\psi_{p m}\right)^{2} \\
& i_{d_{-} \text {south }} \approx \frac{1}{L_{d}}\left(\psi_{d}-\psi_{p m}\right)+\frac{1}{2} \frac{d^{2} i_{d}}{d \psi_{d}^{2}}\left(\psi_{d}-\psi_{p m}\right)^{2}
\end{aligned}
$$

Where injected signal is sinusoidal with given amplitude and frequency, so magnetic flux is given as:

$\psi_{d}=\int u_{d} d t=\int U_{m} \sin \left(\omega_{c} t\right) d t=-\frac{U_{m}}{\omega_{c}} \cos \left(\omega_{c} t\right)$

After substituting (14) into (13), current $i_{d}$ can be written as:

$i_{d}=I_{d \_D C}+I_{d \_c} \cos \left(\omega_{c} t\right)+I_{d \_2 c} \cos \left(2 \omega_{c} t\right)$

Where:

$R_{d}=\frac{1}{L_{d}} ; R_{d}^{\prime}=\frac{1}{2} \frac{d^{2} i_{d}}{d \psi_{d}^{2}}$

$I_{d_{-} D C}=\frac{1}{2} \frac{U_{m}^{2} R_{d}^{\prime}+\psi_{p m}^{2} R_{d}^{\prime} \omega_{c}^{2}-\psi_{p m} R_{d}}{\omega_{c}^{2}}$

$I_{d_{-} c}=\frac{U_{m}\left(2 R_{d}^{\prime} \psi_{p m}-R_{d}\right)}{\omega_{c}} ; I_{d_{-} 2 c}=\frac{1}{2} \frac{U_{m}^{2} R_{d}^{\prime}}{\omega_{c}^{2}}$

The block diagram of PM polarity detection method is shown in Figure 8. The d-axis current signal is processed similarly as q-axis current in case of initial position detection. Amplitude polarity of $2^{\text {nd }}$ harmonic of the injected signal $I_{d \_2 c}$ identifies the polarity of PM. If $I_{d-2 c}>0$, determined position is correct, but 


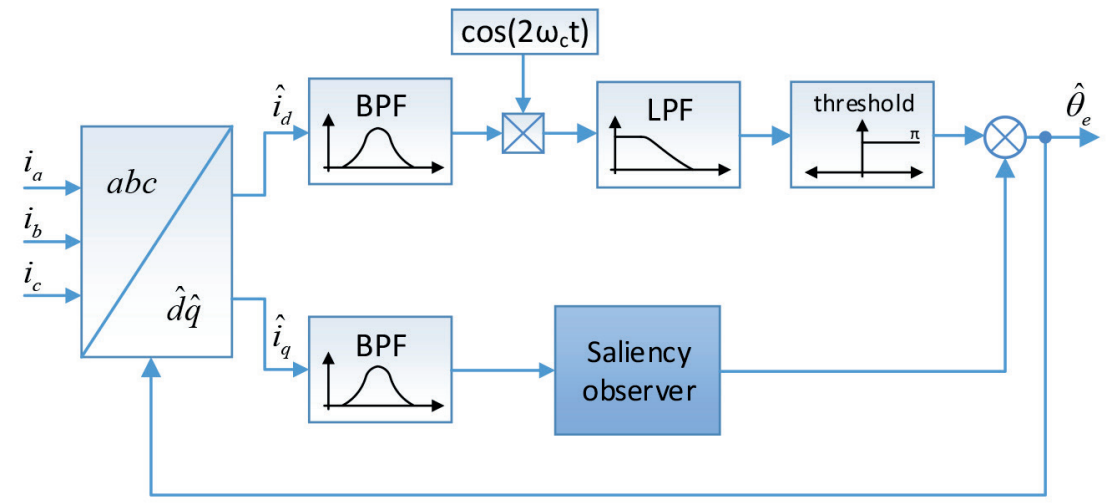

Figure 8 Block diagram of Saliency observer with PM polarity detection algorithm

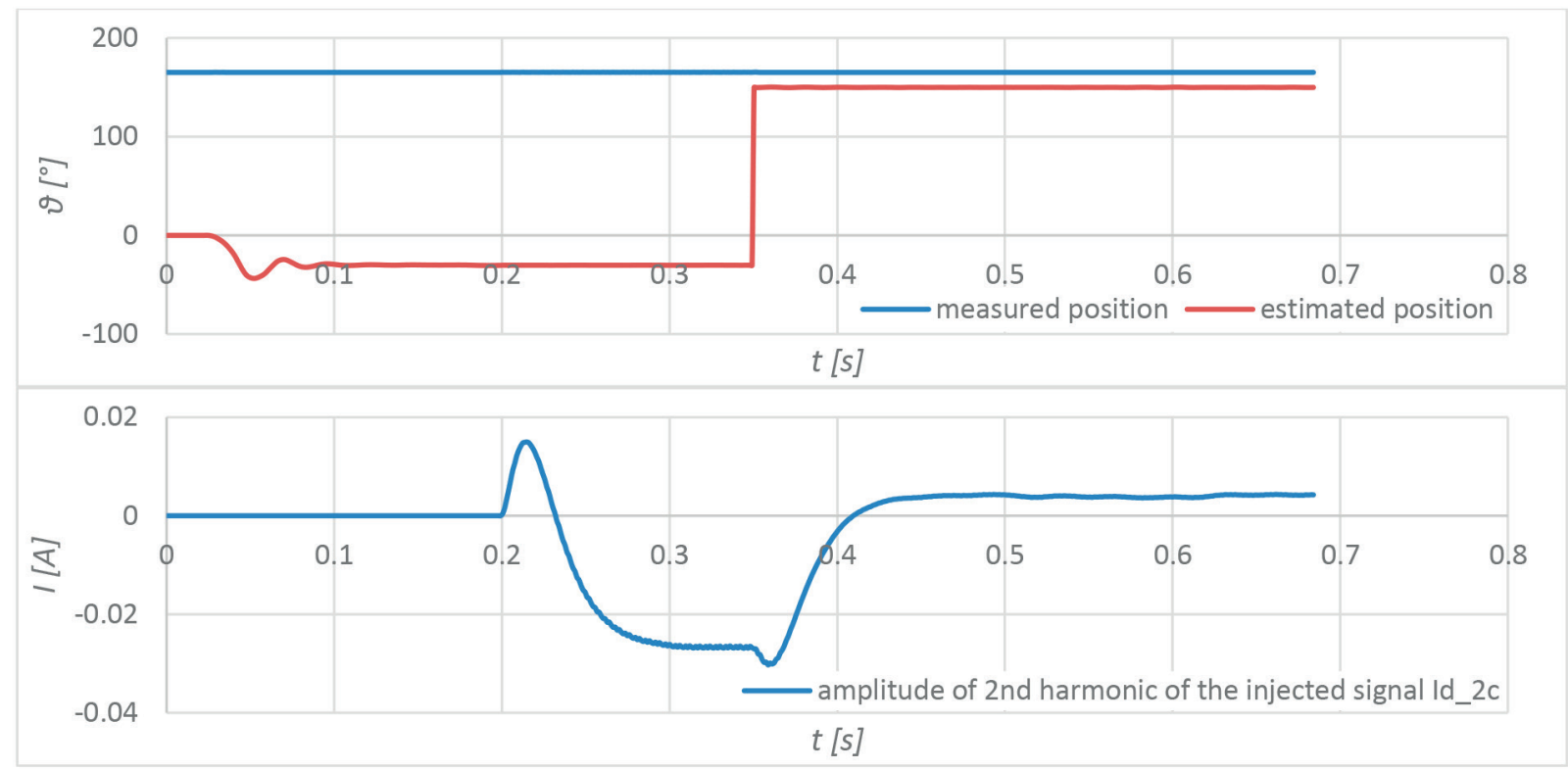

Figure 9 Initial position detection respecting PM polarity

if $I_{d 2 c}<0$, the angle offset $\pi$ has to be added to the estimated position.

Then both algorithms result into one solution for initial position detection respecting the PM polarity and estimation the rotor flux position for zero and small speed. The fusion of both algorithms can be seen in Figure 9.

\section{Experimental Results}

The described self-sensing technique was verified on real PMSM motor. The NXP MPC5643L MCU for automotive and industrial applications was used as control device. The estimated position was compared with position measured by encoder.

Initial position detection including the PM polarity correction can be seen in Figure 9. At the beginning of the test, the south pole of PM is detected. As soon as the negative steady state of the
$I_{d 2 c}$ component is evaluated, the estimated position is corrected by $180^{\circ}$.

In Figure 10 is the PMSM rotating at $300 \mathrm{rpm}$ in negative direction. At $0.28 \mathrm{~s}$, the same speed in positive direction is required. The measured rotor flux position is compared with estimated position in middle graph. Position estimation error in electrical degrees is plotted below. The speed ripple is caused mainly by cogging torque, most visible at low speeds. This issue can be subject of the following research.

\section{Conclusion}

In this paper, the self-sensing control of PMSM at zero and low speed was presented. The high frequency voltage signal was used to excite the motor saliency which is a function of the rotor position. The saliency observer as well as the magnet polarity 

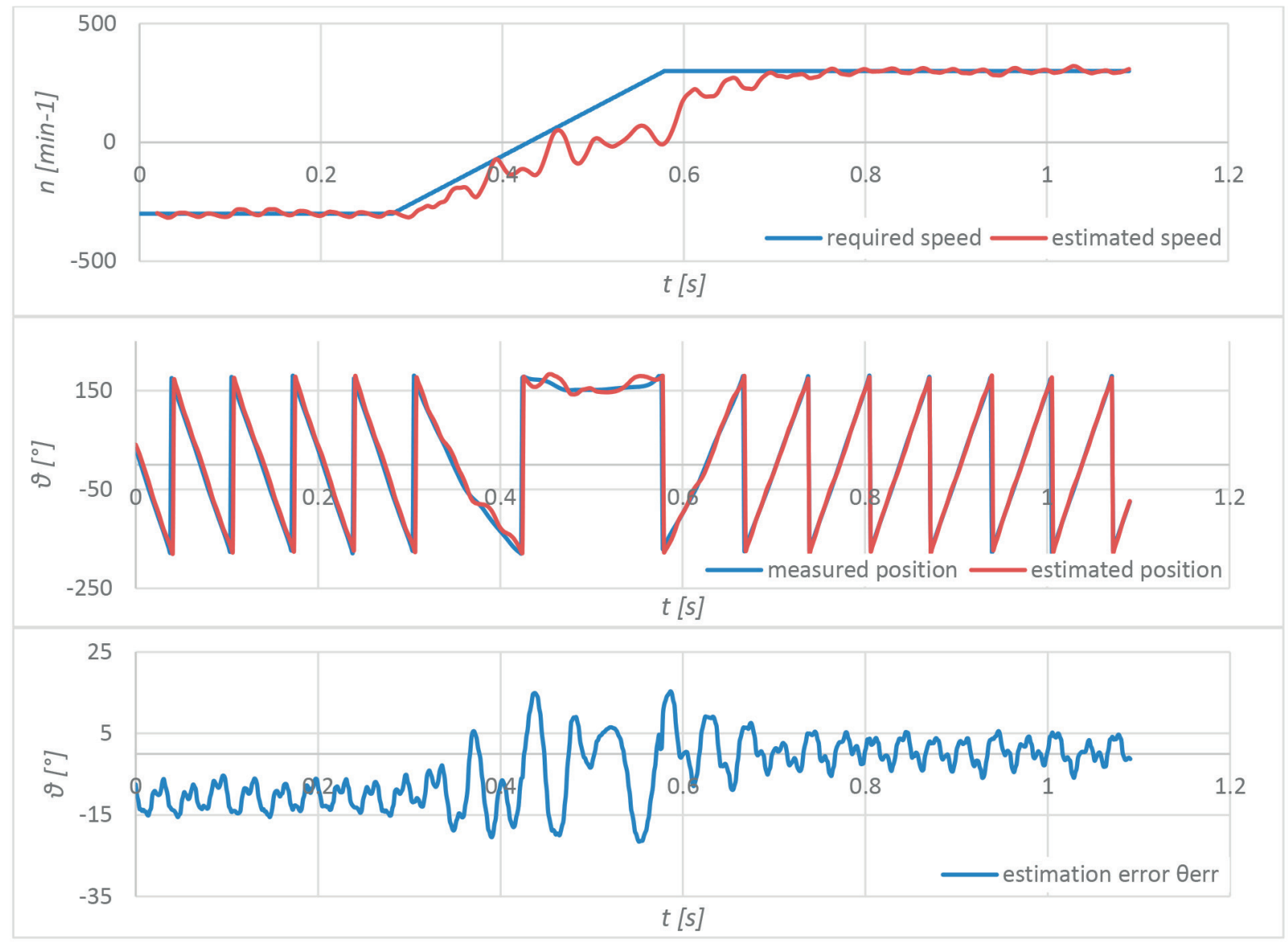

Figure 10 Reversing polarity of required speed

detection method was derived from the motor model considering high frequency signals. Tracking this saliency leads to the rotor flux position which is considered as a rotor position. Proposed algorithms return a correct initial position which enables full start-up torque generation. Both methods were implemented and verified on a real experimental rig with PMSM motor. Achieved results have confirmed the theoretical assumptions and shown that the methods based on the motor anisotropy can be successfully used in self-sensing control.
An additional optimization of the algorithm, accuracy improvement or torque ripple reduction will be a subject of further work.

\section{Acknowledgement}

This work was supported by SRDA No. SK-CN-2015-0007 and the R\&D Operational Program Centre of Excellence of Power Electronics Systems and Materials for their components II. ITMS 26220120046.

\section{References}

[1] Proceedings of International Conference Symposium on Sensorless Control for Electrical Drives. (SLED 2017), Italy, 2017.

[2] CONSOlI, A., SCARCElla, G., SCELBA, G., TESTA, A., TRIOLO, D. A.: Sensorless Rotor Position Estimation in Synchronous Reluctance Motors Exploiting a Flux Deviation Approach. IEEE Transactions on Industry Applications 43(5), 1266-1273, 2007.

[3] SCARLETTA, G., SCELBA, G., TESTA, A.: High Performance Sensorless Controls Based on HF Excitation: A viable Solution for Future AC Motor Drives? In IEEE Workshop on Electrical Machines Design, Control and Diagnosis (WEMDCD), Turkey, 2015.

[4] FILKA, R., BALAZOVIC, P., DOBRUCKY, B.: Transducerless Speed Control with Initial Position Detection for Low Cost PMSM Drives. Proceedings of 13th International Power Electronics and Motion Control Conference (EPE/PEMC 2008), Poland, 2008. 
[5] BOJOI, R., PASTORELli, J., BOTTOMLEY, J., GIANGRANDE, P., GERADA, C.: Sensorless Control of PM Motor Drives - A Technology Status Review. Proceedings of the IEEE 1st Workshop on Electrical Machines Design Control and Diagnosis (WEMDCD (13), France, 168-182, 2013.

[6] CONSOLI, A., SCARCELlA, G., TESTA, A.: Industry Application of Zero-Speed Sensorless control Techniques for PM Synchronous Motors. IEEE Transactions on Industry Applications, 37(2), 513 - 521, 2001.

[7] STUlRajTER, M., HRABOVCOVA, V., FRANKO, M.: Permanent Magnets Synchronous Motor Control Theory. Journal of Electrical Engineering, 58(2), 79 - 84, 2007.

[8] HRABOVCOVA, V., RAFAJdUS, P., FRANKO, M., HUDAK, P.: Measurements and Modeling of Electrical Machines. EDIS Publishing house of University of Zilina, Zilina, 2004.

[9] RIBEIRO, L., DEGNER, W., BRITZ, F., LORENZ, R.: Comparison of Carrier Signal Voltage and Current Injection for Estimation of Flux Angle or Rotor Position. Proceedings of IEEE Industry Applications Conference, USA, 1998. 